\title{
Advances in Human-Automation Collaboration, Coordination and Dynamic Function Allocation
}

\author{
Barrett S. CALDWELL ${ }^{1}$, Megan NYRE-YU and Jordan R. HILL \\ Purdue University, West Lafayette, USA
}

\begin{abstract}
Effective integration of humans and automation in control systems engineering has been an ongoing effort since the original publication of McRuer's descriptions of human operators in servomechanism systems in 1959. Over the past 60 years, increasing capabilities of automation and computer systems have resulted in changing considerations of function allocation and human-automation interaction since Fitts' "Humans are better at / Machines are better at" descriptions of the early 1950s. The processes of distributed autonomy and dynamic function allocation in modern human-automation and human-robotic interactions benefit from increased computing capabilities, resulting in systems with potentially fluid (and sometimes conflicting) boundaries for human vs. automation control. Using examples from human and robotic spaceflight, robotics can demonstrate significant autonomy (automated "safe-moding" and restart by Mars rovers), and humans may have limited autonomy (when astronauts conducting extravehicular activity rely on and wait for ground controllers to create or modify procedures to complete required tasks). Proposed future advances in human-automation interaction and coordination include the development of "centaur" teams of humans interacting with sophisticated software and robotic agents as team members (rather than fixed allocations as human-controlled servos or automationcontrolled autonomous systems). Approaches within the authors' lab include qualitative research of process and cognitive task demands to create functional architecture for AI applications in cyber security. Another method uses agentbased modeling to incorporate individual thinking style and interpersonal interactions in task performance simulations, effectively creating more robust hybrid systems incorporating cognitive and social factors in complex settings.
\end{abstract}

Keywords. Autonomy, Centaur Hybrid Computing, Distributed Expertise, Expertise Dimensions, Human Supervisory Control, Situation Awareness.

\section{Introduction}

Effective integration of humans and automation in control systems engineering has been an ongoing effort since the original publication of McRuer's descriptions of human operators in servomechanism systems in 1959 [1]. Over the past 60 years, increasing capabilities of automation and computer systems have resulted in changing considerations of function allocation and human-automation interaction since Fitts' "Humans are better at / Machines are better at" descriptions of the early 1950s [2]. The processes of distributed autonomy and dynamic function allocation in modern human-

\footnotetext{
${ }^{1}$ Corresponding Author, Email: bscaldwell@purdue.edu.
} 
automation and human-robotic interactions benefit from increased computing capabilities, resulting in systems with potentially fluid (and sometimes conflicting) boundaries for human vs. automation control.

These advances have created, in many ways, a more complex and challenging set of problems in human-automation interactions and complex systems design and integration. Compared to science-fiction dreams of fully automated cities and robotic assistants supporting any and all phases of human endeavor, our automation, artificial intelligence and robotics (A2IR) environment at the end of the second decade of the $21^{\text {st }}$ Century is decidedly deficient. In areas ranging from aviation to cybersecurity to healthcare, A2IR systems and software is neither ready nor capable of functioning effectively or independently in all situations; even more troubling is the struggle between the A2IR agents and humans who still control or manage the systems in which these agents are also components.

Further, hidden within the science-fiction fantasies of robust, successful, and resilient A2IR is a problem engineers recognize in all damped feedback response systems. No engineering system (or sociotechnical system) responds instantaneously or perfectly to any state change input signal. Depending on the system, hysteresis, damping, lag, and other limitations all influence the ability of the engineering system to effectively track and efficiently respond to the required state change [3]. Unfortunately, real-world experiences as well as sociotechnical management simulations have demonstrated that human decision-makers' incorporation of system dynamics constraints in managing complex systems is brittle and subject to a number of cognitive limitations [4], [5] that can be assisted by "preview displays" or other software tools.

As a result of these conflicting pressures, an effective short term solution to the transition to improved A2IR technologies seems to be found not in the selection (or worse yet, competition) between human and automation control of complex systems, but improved system design to enable joint human-automation cooperation and system management. However, improved information flow and shared understanding of intent and function allocation between humans and A2IR technologies requires additional design emphasis on clear and mutually interpretable communication, in essence extending the concepts of shared mental models or shared situation awareness [6], [7] to human-automation partnering.

\section{Discussion}

\subsection{Information to improve awareness and performance}

The integration of information and shared understanding between human and A2IR technologies draws on a variety of disciplines, including cybernetics and information theory as well as human factors engineering. While the origins of information theory as the reduction of entropy associated with message uncertainty have certainly generated tremendous advances in communications engineering and computer science, this aspect of accurate information flow as a technical problem was only one of three linked questions about enabling effective communication; the other questions were semantic and effectiveness problems [8]. According to Shannon and Weaver [8], "the effectiveness problems are concerned with the success with which the meaning conveyed to the receiver leads to the desired conduct on his part" [8, p. 5, emphasis in original]. The mathematical theory of communication, as conceived by Shannon and 
based on Wiener's advances in cybernetics [9], was considered to be a rigorous approach to the technical problem, but only a partial contribution to the effectiveness problem. In essence, then, the discussion of effective human-machine information flow and sharing is an explicit consideration of the effectiveness problem in both directions - not only that the automation as receiver enacts the desired conduct of the human, but that the human as receiver understands and responds appropriately to the meaning conveyed to the human by the automation.

A second, and more recent, tripartite discussion of information processing and sensemaking at the human level is generally known as "situation awareness," a term originally coined by pilots and most frequently associated with task performance and human-machine interactions in the aviation domain [10]. The three levels of situation awareness include: perception of relevant elements of the environment; sensory processing and cognitive comprehension of those elements; and projection of the impact of those elements into the future, with implications for task performance. Importantly, the determination of relevant and meaningful environmental elements is highly domain- and goal-dependent. Echoing the Shannon and Weaver arguments decades earlier, the technical problem of enabling presentation and perception of data from the environment is only a piece of the broader effectiveness problem of comprehension and projection of those data within the context of effective task performance.

These problems are magnified in distributed team performance contexts, where the relevant environmental data and conditions available to one member of a complex systems operations team are not also available in real time to other members of the team. This lack of availability may be due to overall task complexity, differences in operational demands and expertise, large numbers of technical interfaces all requiring distinct skills and attention, or distance and time delays preventing shared synchronous presentation and understanding [11]. The construct of distributed supervisory coordination and distributed expertise emphasizes the effective sharing of expertise and task-related information among team members. Importantly, though the original considerations of distributed supervisory coordination described by Caldwell [11] do emphasize teams of humans, there is no explicit exclusion of sufficiently robust and capable A2IR systems to be able to also function as team members as automation and software agent technologies evolve.

\subsection{Where is the field going?}

Some recent work advancing the frontier of human-automation teaming has begun to consider mutual requirements for information flow between expert humans and advanced software agents. Examples of these human-automation team configurations are described as "centaurs," highlighting the hybrid nature of human expertise collaborating with artificial intelligence computer science design of semi-autonomous agents.

Beginning with examples in deep learning applied to chess, centaur computing teams have developed in a number of areas, ranging from A2IR-supported soldiers to "centaur care teams" for cancer care and other healthcare settings [12], [13]. As described in the discussion on situation awareness, the specific contextual and environmental elements of each application domain will require distinct analysis of both explicit domain knowledge and additional dimensions of human expertise for experts in that task setting [14], and the requirements of learning algorithms and 
training / test set determinations for supporting software agents. There is an additional requirement of effective coordination in time-critical settings, where effective communication and coordination is not simply supporting a general decision problem, but also has task deadlines and temporal requirements that also determine success and failure criteria for performance. As a result, effective centaur teams must not only consider effective allocation of functions and decisions between the human experts and software agents, but how these allocations can be managed dynamically to improve performance within task and time constraints for performance.[3]-[5], [15]. The following sections describe examples of initial analysis approaches and requirements definitions for creating centaur teams in two areas of human-A2IR interactions.

\section{Human-automation interaction and dynamic function allocation}

As technology advances, so to do opportunities to design human-automation interaction in complex environments. However, these require careful consideration in multiple dimensions of work, including cognitive processes, team process behaviors, and effects of task stressors [16]. Research in the authors' lab has sought to address some of these factors across different methods and task environments, described below. While the lab's efforts span multiple project application areas and complex systems implementations, the work is united under a common theme: studying how people get, share, and use information... well. In the context of human interactions with A2IR, the challenge is increased because at least one of the partners sharing information must be explicitly designed and built to provide information in compatible ways, even though our understanding of how humans effectively coordinate implicit and informal information is still limited. In addition, assumptions about normative forms of communication should not be considered universally superior, given combinations of situational, technological, and even neurodiversity considerations of how and what information can be effectively shared [17]-[19].

\subsection{Dimensions of Expertise for Coordinated Function Allocation}

The original configuration of Fitts' List allocations of functions between humans and machines uses a limited conceptualization of expertise to consider relative strengths of humans and automation technologies, focusing primarily on sensorimotor performance and domain-based cognitive and strategic capability [2]. By contrast, group dynamics researchers as early as the 1950s through the 1970s specifically emphasized coordinated teamwork as an integration of task-based skills and socioemotional, or interpersonal, performance [18]. In order to demonstrate effective task-related and cohesive group behaviors, expert team members were expected to appropriately balance multiple interactive skills; these skills have been since developed into four software simulation module classes to demonstrate team interaction processes (See Table 1) [18]. 
Table 1. Descriptions of Expert Team Coordination Processes, from [18].

\begin{tabular}{ll}
\hline $\begin{array}{l}\text { Expertise Coordination } \\
\text { Process }\end{array}$ & Descriptions of Coordination Processes \\
\hline Asking & $\begin{array}{l}\text { Novices (or team members acting outside of their area of expertise) bring } \\
\text { queries to the team; queries are then available to be answered by one or } \\
\text { more experts, depending on complexity, comprehensibility, expert } \\
\text { availability, and query understanding }\end{array}$ \\
\hline Learning & $\begin{array}{l}\text { Team members are socialized as part of an expert team, and use existing } \\
\text { experts and reference sources to develop expertise in a particular area } \\
\text { while learning about the structure and processes of the team }\end{array}$ \\
\hline Sharing & $\begin{array}{l}\text { A mixed group of novices and experts interact using shared information } \\
\text { tools (such as a discussion list or chat room) to exchange information, } \\
\text { perspectives, and social affiliation, in addition to specific task oriented } \\
\text { discussions }\end{array}$ \\
\hline Solving & $\begin{array}{l}\text { Members of the expert team are responsible for monitoring and } \\
\text { troubleshooting problems and are focused on effective task performance to } \\
\text { maintain system functioning }\end{array}$ \\
\hline
\end{tabular}

These configurations, as well as other research describing dimensions of expertise, emphasize the importance of effective team coordination as incorporating both individual skills (beyond one's specific domains of declarative knowledge) and interpersonal skills. The exploration of dimensions of expertise [14] lays out these individual / interpersonal complementary skills, as well as task / team complementary skills, as a visual display of multiple combinations of expertise types (See Figure 1).

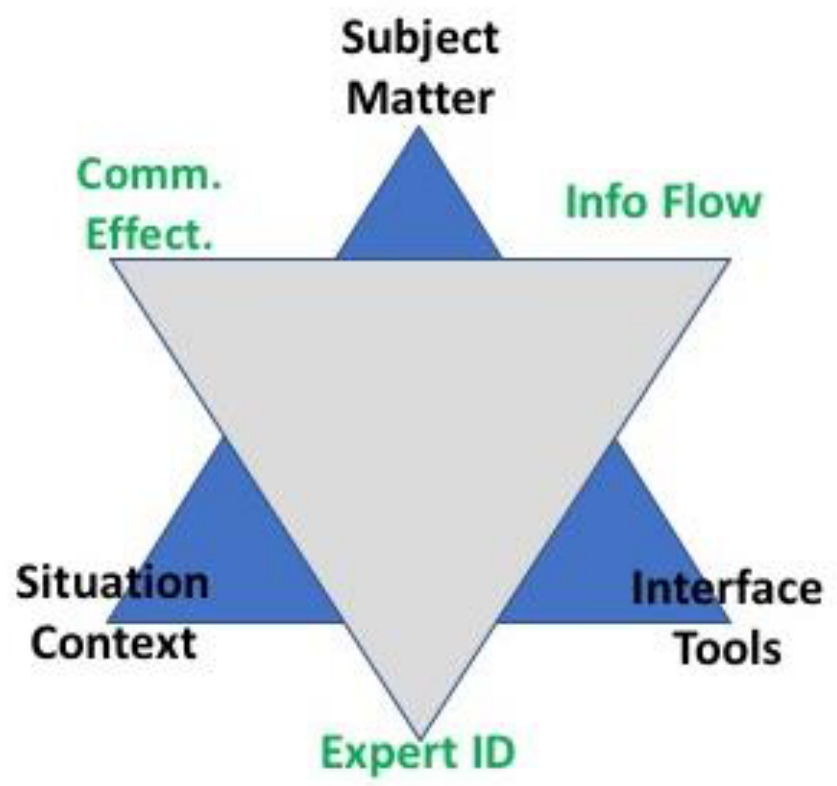

Figure 1. Six Dimensions Of Expertise Integrating Individual (Triangle Point Up) And Interpersonal (Triangle Point Down) Expertise Coordination Requirements. Adapted from [14]. 
Additional indications of the need for expanding these interpersonal expertise coordination dimensions among humans are shown in the following emerging domains of human-robotic cooperation.

\subsection{Human-robotic cooperation for human spaceflight exploration}

Robotic scientific operations have occurred on Mars since the 1970s; first with landers and then with rovers in 1997. The National Aeronautics and Space Administration has successfully landed and operated four different rovers on Mars: Sojourner, Spirit and Opportunity (the twin Mars Exploration Rovers), and Curiosity (the Mars Science Laboratory) [20]-[22]. Due to the facts that rovers are mobile, there is a 4-22 minute one-way light time delay on all communications between Earth and Mars, and that there are only a few downlink and uplink opportunities each day with which to communicate with robotic assets on Mars [21], rovers have certain levels of autonomy when it comes to the performance of some activities (and very little autonomy with others). For example, rovers are not "driven" on the Martian surface like a remotecontrolled vehicle - the time delay on commands makes such a mobility model impossible. Instead, Rover Drivers give waypoints and end state commands to the rover and then the rover uses stereo images and other wayfaring technologies to avoid obstacles to reach the desired destination [20], [23]. Contrarily, when it comes to scientific activities, a Mars rover has a low level of autonomy; a large group of scientists on Earth choose scientifically interesting targets and the associated tasks for the rover, and rover engineers ensure that those science activities can be feasibly performed while maintaining the safety of the rover [24].

Currently, there are plans in the space exploration community to send humans to Mars [25], [26]. In order to support human life on Mars, astronauts will need to perform many extravehicular activities (EVAs) — where a suited astronaut leaves the safety of the spacecraft_-due to the operational flexibility that EVA provides [27] and despite the fact that EVA poses higher risk to astronauts than nearly any other activity in space [28]. Currently, EVAs are performed with small crews being supported by a large group of experts on Earth in the Mission Control Center. The personnel in Mission Control are responsible for processing and monitoring the majority of the necessary information streams and keeping the astronauts safe during EVA [29], [30]. There is evidence that this current model of EVA is incompatible with the time-delayed communication constraints and the communication blackout periods that come with human deep-space missions [31], and is also not scalable to situations where they may be more than one EVA being performed simultaneously [15].

Current deep-space human mission architectures plan for small crew sizes of 3-6 astronauts [32], [33], meaning that sending larger crews on deep-space missions to compensate for the lack of Earth-based support is not being considered. Due to this, the complex systems required to keep humans alive in deep-space will quickly overwhelm unaided crews [31]; the first and third author of this paper have observed the overabundance of information monitoring and processing requirements for small crew in a Mars analog setting, which supports this assertion [34]. Therefore, taking advantage of increases in computational capabilities in the decades since the beginning of space exploration, the development of automated support systems to monitor and process critical information and aid in decision-making will be necessary. With the goal in aiding automation development, both [35] and [36] characterized the EVA work 
domain using the principles of Cognitive Work Analysis [37] in order to develop an automated path-planning tool and a decision support system framework, respectively.

The third author of this paper is looking to identify the information monitoring/processing requirements for the performance of scientific EVA on Mars. The goals of this work are to be able to identify the necessary information streams, how the information is collected and delivered to the astronauts, and the necessary actions that need to be taken with the specified information. This is being done through qualitative interviews with rover scientists and engineers in order to be able to take the knowledge gained through experience with robotic exploration and apply it to human exploration. Once there is a clearer picture regarding the necessary information streams for scientific EVA, automated support systems can be developed, ensuring that all developed tools not only perform their roles acceptably, but that each individual tool functions well with other automated support systems, and the astronauts they are designed to support. Dynamic function allocation will be important in this application, as it will be of the utmost importance to ensure that the astronauts are kept in-the-loop as to the current actions of the automation and can take over the functions of the robotic aid if necessary.

\subsection{Human-automation team design using simulation}

Another approach in the authors' lab explores the idea of teams of humans and automation working together through simulation research. The goal of this research thrust is to establish better understanding of relationships between social and cognitive factors, and how these affect performance in different configurations of teams (including human and non-human entities). Onken [38] created a foundation for building these computational models. His doctoral work incorporated team member attributes in task performance for NASA mission control settings. This work explored event response, and advanced towards understand needed expertise and attributes to improve performance. Master's thesis research by the second author expanded upon Onken's work to build further understanding of attribute dynamics in task performance using agent-based modeling (ABM) [39].

Simulation methods employing advanced computing capabilities are able overcome time and resource boundaries in the real-world [40]. Moreover, these methods can accommodate experimental research in human-automation teaming by creating virtual teams from real team data and testing different attribute levels and combinations in a simulated environment. Though only a prototype of how ABM simulation methods can explore this area, the research paves a path to studying complex sociotechnical systems through computational approaches, more specifically in how components of a team (human-human, human-robot, or robot-robot) work together to complete a task [41]. This type of research also allows for experimentation in dynamic function allocation and how it affects overall performance.

The flexibility and power afforded by modern-day computing capabilities allow for faster exploration of the domain and clearer identification of emergent patterns and factor interactions. With respect to dynamic function allocation, this research illustrates how computing opens a new avenue for understanding and designing humanautomation teams, and identifying needs for balancing member attributes in a variety of task settings. 


\subsection{Cyber security centaurs}

Cyber security is a rapidly evolving domain in which technology is advancing to meet demands of defenders. More specifically computer security incident response involves humans and automation ingesting large amounts of data, detecting threats, and taking action to mitigate them, all in a matter of seconds. The field is fast arriving to the conclusion that, in order to adequately defend a given network, automation in more advanced forms (i.e. machine learning and artificial intelligence) is a necessary component of incident response. 'Cyber security centaurs' have been proposed in business literature as the next step for the field [42], and newly-released automation and orchestration platforms are integrating human expertise into data analysis and process execution [43]. These ideas pivot on the concept the humans and technology will become teammates, working together to detect and mitigate cyber threats. Yet, as this technology advances in machine learning capability and computing speed, the future role and strategic development of the human component remains undefined.

The authors posit that dynamic function allocation will soon become a topic of interest as artificial intelligence grows in both proficiency and popularity in cyber security. Current automation remains at the lower tiers of incident response, with supervised learning and some monitored task execution. Considering the pain felt in many computer security organizations is not enough skilled workers [44], automation is seen as the solution to one of the biggest problems currently experienced in the field. However, the next stages of development and integration of automation into higherskill tasks depends on better understanding of human-automation interaction in this fast-moving, decision-sensitive domain.

Specialized technology design is often preceded by achieving better understanding of how the tasks are currently performed, and what requirements are needed in order to perform them well [3], [45], [46]. Cyber security analyst jobs are considered knowledge work [47], indicating that expertise is central to performing the job well. Dissertation work by the first author explores dimensions of expertise [14] beyond merely subject matter in the context of computer security incident response to better define technology requirements in relation to the tasks currently performed. Methods are based in qualitative research to accurately collect information on the analysts and tasks directly in the environments in which they work. Studying teams in different sectors with varying levels of technology to assist analysts also gives ranges of maturity and needs to widen the scope of potential customizations or automation settings. An additional study elicits knowledge from cyber security experts to better understand holistic expertise needs for lower tier tasks and transitions (or escalations) between novices and experts. Finally, comparing the findings of both methods to current development in automation will help draw attention to future areas of investigation when translating expertise from humans to machines, and striking a balance between the two for human-machine teaming.

\section{Conclusions}

The changing landscape of automation and machine learning technologies suggests that the Fitts list of human-machine function allocation from the 1950s is no longer accurate, but fundamentally insufficient, for examining new challenges of human-automation interactions and dynamic function allocation for complex task scenarios. Thus, a full 
re-presentation of the Fitts list is beyond the scope of this paper, both in terms of current automation capabilities and the limitation of FItts' considerations of sensorimotor and cognitive expertise. Centaur teams in space and on Earth will, for the foreseeable future, require coordination and mutual support integrating humans and machines in the face of dynamic challenges, rather than fixed allocations that pit those resources against each other.

However, it is possible to consider domains of needed improvements in humanautomation teaming based on the six dimensions of expertise framework presented in Figure 1 above. Again, a full exploration of machine learning and social "chatbot" interactions is beyond the scope of this paper, and is itself a daunting challenge to address in the face of a rapidly advancing technology frontier (see [48-49] for popular press descriptions as of summer 2019). It is not envisioned that machine learning and automation capabilities currently demonstrated fully embodies any of the six dimensions of expertise presented in [14], let alone a single functional integration effectively working with humans in a specific centaur application, as of 2019. However, a simple overview of needed capabilities is presented, based on the authors' initial considerations (rather than an exhaustive literature review), in Table 2. Extensions from general social media or popular "chatbot" human-computer interactions are problematic, since the interaction goals of Alexa, Siri or similar tools are different from a true human-agent centaur task performance teammate. (Ironically, it is reported that Alexa's responses to queries about machine learning include "But I don't know a lot about it, to be honest." [48]).

Table 2. Simple overview of needed capabilities.

\begin{tabular}{ll}
\hline $\begin{array}{l}\text { Expertise Dimension } \\
\text { (from [14]) }\end{array}$ & $\begin{array}{l}\text { Current Machine Learning Capabilities and Limitations (Authors' } \\
\text { Opinion Only) }\end{array}$ \\
\hline Subject Matter Domain & $\begin{array}{l}\text { IBM Watson has demonstrated champion-level general topic knowledge in } \\
\text { the gameshow "Jeopardy"; however, social media chatbots still focus on } \\
\text { popular topics rather than integrative subject matter. }\end{array}$ \\
\hline Interface Tools & $\begin{array}{l}\text { Increasing expertise in terms of accessing recognized multimedia, } \\
\text { documents, or general knowledge interfaces. Interactions with real-world } \\
\text { objects in unconstrained and human-risky environments, such as } \\
\text { roadworthy vehicles and automated guided robots in factories, are still of } \\
\text { limited autonomy and capability as of mid-2019. }\end{array}$ \\
\hline
\end{tabular}

Situation Context Pre-identified contexts are largely recognized through pattern recognition and guided training sets. Machine learning tools still have significant problems with acknowledging and combatting biases and misinterpretations based on training set contexts.

\begin{tabular}{ll}
\hline Expert Identification & $\begin{array}{l}\text { Machine learning tools can identify explicitly defined roles and primary } \\
\text { expertise designations of human team members; experience-based } \\
\text { recognition of implicit capabilities or past knowledge is limited to } \\
\text { aggregated training of agent experience with those team members. }\end{array}$ \\
\hline $\begin{array}{l}\text { Information Flow Path } \\
\text { Support }\end{array}$ & $\begin{array}{l}\text { Dynamic monitoring and "quality of service" enhancement within a specific } \\
\text { medium has improved greatly in audio, video, and word recognition for text } \\
\text { messaging. shifting of communication across flow paths (communication } \\
\text { channels or media) very limited. }\end{array}$ \\
\hline Communication & $\begin{array}{l}\text { Weak in terms of dynamic, Turing-style conversation management, but } \\
\text { human-aided "bots" have demonstrated capabilities in misinformation and } \\
\text { social influence campaigns. Collaborative problem solving and sharing } \\
\text { (see [18]) on dynamic tasks varies greatly by problem domain. }\end{array}$ \\
\hline
\end{tabular}


In closing, work from the authors' lab seeks to apply multi-dimensional and multidisciplinary frameworks to achieve better understanding of human needs and to translate these into system requirements for automation design and dynamic function allocation. The design of human-automation centaurs represents a distinct, yet challenging, approach both to enhancing A2IR capabilities and retaining the benefits of human expertise. In contexts ranging from cybersecurity to spaceflight to healthcare, transportation, and other domains, cooperative integration rather than antagonistic conflict provides an important enabling capability for the extended transitions from fully manual to fully automatic management of complex sociotechnical systems.

\section{Acknowledgement}

Portions of this work have been supported by the Purdue University Faculty Scholars Program, NASA Planetary Science and Technology Through Analog Research (PSTAR) Program grant "Biologic Analog Science associated with Lava Terrains (BASALT)" and NASA Solar System Exploration Research Virtual Institute (SSERVI) Program grant "Field Investigations to Enable Solar System Science and Exploration (FINESSE"). Barrett Caldwell was the Purdue Principal Investigator for both grants (FINESSE: NNX14AF37A; BASALT: NNX15AM05A).

\section{References}

[1] D.T. McRuer and E.S. Krendel, The human operator as a servo system element, J. Franklin Inst., vol. 267 , no. 5, pp. 381-403, 1959 .

[2] J. C. F. de Winter and D. Dodou, Why the Fitts list has persisted throughout the history of function allocation, Cogn. Technol. Work, vol. 16, no. 1, pp. 1-11, 2014.

[3] B.S. Caldwell, Knowledge sharing and expertise coordination of event response in organizations, Appl. Ergon., vol. 39, no. 4, pp. 427-438, 2008.

[4] B.S. Caldwell, Cognitive Challenges to Resilience Dynamics in Managing Large-Scale Event Response, J. Cogn. Eng. Decis. Mak., vol. 8, no. 4, pp. 318-329, 2014.

[5] B.S. Caldwell, Framing, Information Alignment, and Resilience in Distributed Human Coordination of Critical Infrastructure Event Response, Procedia Manufacturing, vol. 3, pp. 5095-5101, 2015.

[6] J.C. Gorman, N.J. Cooke and J.L. Winner, Measuring team situation awareness in decentralized command and control environments, Ergonomics, vol. 49, no. 12-13, pp. 1312-1325, Oct. 2006.

[7] R.J. Stout, J.A. Cannon-Bowers, E. Salas and D.M. Milanovich, Planning, Shared Mental Models, and Coordinated Performance: An Empirical Link Is Established, Hum. Factors, vol. 41, no. 1, pp. 61-71, Mar. 1999.

[8] C. E. Shannon and W. Weaver, The Mathematical Theory of Communication, The University of Illionis Press, Urbana, 1949.

[9] N. Wiener, Cybernetics: or Control and Communication in the Animal and the Machine. The MIT Press, Cambridge, 1948.

[10] M.R. Endsley, Theoretical Underpinnings of Situation Awareness: A Critical Review, in M.R. Endsley and D.J. Garland (eds.) Situation awareness: analysis and measurement, Lawrence Erlbaum Associates, Mahwah, 2000.

[11] B.S. Caldwell, Analysis and modeling of information flow and distributed expertise in space-related operations, Acta Astronautica, vol. 56, pp. 996-1004, 2005.

[12] P. Franaszczuk, Intelligent Teams, in A. Kott, K. Choi, B. Forch, P. Franaszczuk, S. Karna, S. Lee, J. Mait, P. Reynolds, B. Sadler, A. Swami, and B. West (eds.) Potential Science and Technology Game Changers for the Ground Warfare of 2050: Selected Projections Made in 2017, Army Research Laboratory, Adelphi, 2018, pp. 22-24.

[13] I. M. Goldstein, J. Lawrence and A. S. Miner, Human-Machine Collaboration in Cancer and Beyond: The Centaur Care Model, JAMA Oncol., vol. 3, no. 10, pp. 1303-1304, 2017. 
[14] S. K. Garrett, B. S. Caldwell, E. C. Harris, and M. C. Gonzalez, Six dimensions of expertise: a more comprehensive definition of cognitive expertise for team coordination, Theor. Issues Ergon. Sci., vol. 10, no. 2, pp. 93-105, Mar. 2009.

[15] B.S. Caldwell and J.D. Onken, Modeling And Analyzing Distributed Autonomy For Spaceflight Teams, in 41st international Conference on Environmental Systems, 2011, https://doi.org/10.2514/6.2011-5135.

[16] H. M. Cuevas, S. M. Fiore, B. S. Caldwell, and L. Strater, "Augmenting team cognition in humanautomation teams performing in complex operational environments," Aviat. Sp. Environ. Med., vol. 78, no. 5, Section II, pp. 63-70, 2007.

[17] L.H. Taha and B.S. Caldwell, Social isolation and integration in electronic environments, Behav. Inf. Technol., vol. 12, no. 5, pp. 276-283, 1993.

[18] B. S. Caldwell, Digital Human Modeling of Behaviors and Interactions in Teams, in V. Duffy (ed.) Handbook of Digital Modeling, 2008 ch. 47.

[19] B.S. Caldwell, Connection, Coupling, and Persistence in Online Social Networks, in D. Haftor and A. Mirijamdotter (eds.) Information and Communication Technologies, Society and Human Beings: Theory and Framework (Festschrift in honor of Gunilla Bradley), IGI Global, Hershey, 2011, pp. 346354.

[20] A. Mishkin, Sojourner: An Insider's View of the Mars Pathfinder Mission. The Berkley Publishing Group, New York, 2003.

[21] S.M. Perl, A Multi-Level Approach to Enhance Information Exchange for the 2011 Mars Science Laboratory Mission, Purdue University, 2011.

[22] K. Tate, Mars Explored: Landers and Rovers Since 1971 (Infographic), https://www.space.com/12404mars-explored-landers-rovers-1971.html, accessed July 10, 2019.

[23] M.W. Maimone, P.C. Leger and J.J. Biesiadecki, Overview of the Mars Exploration Rovers' Autonomous Mobility and Vision Capabilities, in IEEE International Conference on Robotics and Autonomation, http://citeseerx.ist.psu.edu/viewdoc/download?doi=10.1.1.212.2421\&rep=rep1\&type=pdf.

[24] A. Mishkin, D. Limonadi, S.L. Laubach, and D.S. Bass, Working the Martian Night Shift: The MER Surface Operations Process, IEEE Robotics and Automation Magazine, vol. 13, no. 2, pp. 46-53, 2006.

[25] National Aeronautics and Space Administration, Journey to Mars Overview. https://www.nasa.gov/content/journey-to-mars-overview. Accessed Apr 04, 2018.

[26] D. Newman and M. Barratt, Life support and performance issues for extravehicular activity (EVA), in S. Churchill and O. Heinz (eds.) Fundamentals of Space Life Sciences, Krieger Publishing Company, Malabar, 1997.

[27] M.J. Miller, K.M. McGuire and K.M. Feigh, Decision Support System Requirements Definition for Human Extravehicular Activity Based on Cognitive Work Analysis, J. Cogn. Eng. Decis. Mak., vol. 11, no. 2, pp. 136-165, 2017.

[28] N. Wognum, C. Bil, F. Elgh, M. Peruzzini, J. Stjepandić and W.J.C Verhagen, Transdisciplinary systems engineering: implications, challenges and research agenda, International Journal of Agile Systems and Management, Vol. 12, 2019, No. 1, pp. 58-89.

[29] J.D. Onken and B.S. Caldwell, Modeling Mission Operations Trade Spaces and Lunar C3I Capabilities, International Conference on Environmental Systems, 2009, https://www.sae.org/publications/technicalpapers/content/2009-01-2426/.

[30] B. Woolford, W.E. Sipes and E.R. Fiedler, Human Space Flight, in G. Salvendy (ed.) Handbook of Human Factors and Ergonomics, 2012, pp. 910-927.

[31] A. Mishkin, Y. Lee, D. Korth, and T. LeBlanc, Human-Robotic Missions to the Moon and Mars: Operation Design Implications, IEEE Aerospace Conference, 2007, DOI: 10.1109/AERO.2007.352960.

[32] S.J. Hoffman and D.I. Kaplan, Human Exploration of Mars: The Reference Mission of the NASA Mars Exploration Study Team, NASA Johnson Space Center, Houston, 1997.

[33] J.-M. Salotti, R. Heidmann and E. Suhir, Crew size impact on the design, risks and cost of a human mission to Mars, IEEE Aerospace Conference, 2014, DOI: 10.1109/AERO.2014.6836241.

[34] J.R. Hill and B.S. Caldwell, Toward better understanding of function allocation requirements for planetary EVA and habitat tasks, Human Factors and Ergonomics Society 2018 International Annual Meeting, Philadelphia, PA: SAGE Publications, 2018, https://doi.org/10.1177/1541931218621007.

[35] J.J. Marquez, Human-Automation Collaboration: Decision Support for Lunar and Planetary Exploration, $\mathrm{PhD}$ thesis, Massachussetts Institute of Technology, 2007.

[36] M.J. Miller, Decision Support System Development for Human Extravehicular Activity, PhD thesis, Georgia Institute of Technology, 2017.

[37] K.J. Vicente, Cognitive Work Analysis: Toward Safe, Productive, and Healthy Computer-Based Work. Lawrence Erlbaum Associates, Mahwah, 1999.

[38] J.D. Onken, Modeling Real-Time Coordination of Distrubuted Expertise and Event Response in NASA Mission Control Center Operations, PhD thesis, Purdue University, 2012. 
[39] M.M. Nyre, Developing agent-based simulation models of task performance of cognitively diverse teams, MSc thesis, Purdue University, 2016.

[40] M. Nyre-Yu and B.S. Caldwell, Using Simulation Modeling Methods to Study Teams Doing Taskwork, Proc. Hum. Factors Ergon. Soc. Annu. Meet., vol. 62, no. 1, pp. 788-792, Sep. 2018.

[41] M. Nyre-Yu and B.S. Caldwell, Supporting Advances in Human-Systems Coordination through Simulation of Diverse, Distributed Expertise, Systems, vol. 6, no. 4, 2018, doi:10.3390/systems6040039.

[42] S. Truvé, Machine Learning in Cyber Security: Age of the Centaurs, Sommerville, MA, Jan. 2017, https:/www.brookcourtsolutions.com/wp-content/uploads/2017/07/Machine-Learning-in-CyberSecurity-White-Paper-Brookcourt.pdf.

[43] C. Bedell, Definitive Guide to SOAR. CyberEdge Press, Annapolis, 2019.

[44] Bureau of Labor Statistics, Information Security Analysts, Occupational Outlook Handbook, 2016. https://www.bls.gov/ooh/computer-and-information-technology/information-security-analysts.htm. Accessed: Oct 11, 2017.

[45] T.S. Sheridan, Tasks, Errors, and Mental Models, in L.P. Goodstein, H.B. Andersen and S.E. Olsen, (eds.) Task, errors, and mental models: a Festschrift to celebrate the $60^{\text {th }}$ birthday of professor Jens Rasmussen, Taylor \& Francis, Bristol, 1988, pp. 149-160.

[46] P. Mears, Quality improvement tools \& techniques. McGraw-Hill, New York, 1995.

[47] T.H. Davenport, Thinking for a Living, Notes, vol. 18, no. 4, pp. 599-603, 2005.

[48] A. Fell, Alexa, Let's Chat, UC Davis Magazine, Spring/Summer 2019, pp 22-25.

[49] A. Følstad and P.B. Brandtzaeg, Chatbots and the new world of HCI. Interactions, vol. 24, no. 4, pp. $38-42,2017$. 